\title{
Etude comparée de la solubilité et de la viscosité des solutions de caséinate et paracaséinate de sodium en présence de calcium
}

\author{
J.J. Baumy et G. BrulÉ \\ I.N.R.A., Laboratoire de Recherche de Technologie laitière \\ 65 , rue de Saint-Brieuc - 35042 Rennes cedex, France
}

\begin{abstract}
Résumé
Les interactions en milieu aqueux entre caséinate de sodium, paracaséinate de sodium et calcium ainsi que leurs conséquences sur la solubilité et la viscosité ont été étudiées. La solubilité différente de ces deux produits dépend de la teneur en calcium des solutions, du $\mathrm{pH}$ et de la force ionique.

La quantité maximale de calcium fixé aux protéines avant précipitation à $\mathrm{pH} 6,6$ est égale à 7,8 et 5,5 moles par mole de caséinate et paracaséinate (teneur en protéines : $80 \mathrm{~g} / 1$ - force ionique initiale : 0,036). L'abaissement du $\mathrm{pH}$ de 6,6 à 5,4 entraîne une diminution des quantités de calcium fixé. L'augmentation de la force ionique n'a pas d'influence significative sur la quantité de calcium fixé par le paracaséinate, par contre elle entraîne une diminution du calcium fixé par le caséinate.

Le comportement rhéologique des caséinates et paracaséinates en solution à teneurs calciques croissantes est différent : après une légère chute de viscosité mesurée à la même vitesse de cisaillement de $517 \mathrm{~s}^{-1}$ pour les deux produits, on assiste à une augmentation brutale de celle-ci pour le paracaséinate alors que dans le cas du caséinate la viscosité augmente modérément puis diminue progressivement. Une baisse $\mathrm{du} \mathrm{pH}$ ou une augmentation de force ionique modifient ces comportements.
\end{abstract}

Mots clés : Caséinate - Paracaséinate - Calcium - Solubilité - Viscosité.

\section{Introduction}

Les éléments minéraux du lait, bien que quantitativement peu importants, jouent un rôle essentiel tant au plan physico-chimique que nutritionnel et technologique.

Les caséines du lait sont associées en une structure micellaire constituée d'un ensemble de sous-unités appelées submicelles reliées les unes aux autres par du phosphate de calcium (SснмIDT, 1982). Le lait de vache renferme $1,2 \mathrm{~g} / 1$ de calcium, répartis en $0,8 \mathrm{~g} / 1$ fixés aux micelles de caséines et $0,4 \mathrm{~g} / 1$ sous forme soluble associé prin- 
cipalement au citrate et phosphate (PyNe et Mc GANN, 1960). La répartition du calcium entre fractions micellaire et soluble est sous la dépendance d'équilibres complexes faisant intervenir les caséines, le phosphate, le citrate; ceux-ci étant fonction du $\mathrm{pH}$, de la force ionique (BRUlé et al., 1974) et de la température (PIERRE et BRulé, 1981).

La chélation du calcium par les caséines se réalise essentiellement au niveau des résidus phosphoséryles (PARKER et DALGLeIsH, 1981) ; les groupements carboxyliques interviennent aussi (ONo et al., 1976) mais avec une constante d'association 20 fois moindre (SwaIsGOOD, 1982). Les 2 acidités du phosphate de la phosphosérine correspondent à des énergies de liaison différentes (PIERRE et al., 1983).

La stabilité des micelles de caséine en milieu phosphocalcique est étroitement dépendante de la présence de caséine $\chi$; l'hydrolyse de cette dernière sous l'action d'enzymes protéolytiques entraîne la destabilisation des micelles et la coagulation du lait. La solubilité des paracaséines est beaucoup plus faible que celle des caséines (Nitschmann et LehmanN, 1947).

La maîtrise des propriétés fonctionnelles des caséinates et dérivés est étroitement liée à la connaissance des interactions des protéines avec le milieu solvant, dont la fraction minérale, et de leurs conséquences sur la solubilité, les propriétés émulsifiantes et gélifiantes.

Nous nous sommes proposés, dans cette étude, de comparer les interactions caséinate-calcium et paracaséinate-calcium et d'en déterminer les effets sur la solubilité et le comportement rhéologique de ces protéines.

\section{Matériel et méthodes}

Caséinate : le caséinate utilisé était d'origine industrielle (ArmorProtéines) obtenu à partir de lait par précipitation lactique et neutralisation à la soude. Ce produit avait, par $\mathrm{kg}$ de poudre, un extrait sec de $950 \mathrm{~g}$, une teneur en protéines $(\mathrm{N} \times 6,38)$ de $910 \mathrm{~g}$ et des teneurs minérales de $0,7 \mathrm{~g}$ en calcium et $14,2 \mathrm{~g}$ en sodium. Ce caséinate était solubilisé à l'aide d'un mixer Sorvall dans de l'eau bidistillée. Une addition de $0,4 \mathrm{~g} / \mathrm{l}$ d'azoture de sodium aux solutions, dont les teneurs en protéines variaient de 7 à $111 \mathrm{~g} / 1$, permettait d'éviter un développement microbien.

Paracaséinate : le paracaséinate était obtenu à la suite d'une hydrolyse par la présure de solutions de caséinate $(2 \mathrm{ml} / 1$ de présure commerciale diluée 100 fois). A température ambiante $\left(20^{\circ} \mathrm{C}\right)$ et $\mathrm{pH}$ 6,6 la réaction était complète en $2 \mathrm{~h}$. Ces paracaséines étaient utilisées immédiatement après préparation pour limiter l'hydrolyse des protéines autres que la caséine $\chi$. 
Mode d'acidification : l'acidification lente de $\mathrm{pH}$ 6,6 à $\mathrm{pH}$ 6,0 et 5,4 , des solutions contenant $80 \mathrm{~g}$ de caséinate ou de paracaséinate par 1 était obtenue par hydrolyse totale de glucono- $\delta$-lactone. La quantité à ajouter pour obtenir le $\mathrm{pH}$ désiré en $4 \mathrm{~h}$ était respectivement de 0,4 et $0,9 \mathrm{~g} / 1$.

Préparation des échantillons : les échantillons étaient obtenus par addition aux solutions de caséinate et paracaséinate d'une solution de chlorure de calcium (teneur de 17 à $110 \mathrm{~g} / \mathrm{l}$ adaptée à chaque essai pour obtenir une dilution minimale du produit final). Les modifications de force ionique $(\mu)$ étaient réalisées par addition de chlorure de sodium. La baisse du $\mathrm{pH}$ consécutive à l'ajout de $\mathrm{CaCl}_{2}$ était corrigée par de l'hydroxyde de sodium (N). On laissait l'équilibre de complexation se réaliser pendant $1 \mathrm{~h}$ à température ambiante $\left(20^{\circ} \mathrm{C}\right)$.

Fractionnement des échantillons : la fraction soluble des différentes solutions était obtenue par centrifugation (20 min - $1000 \mathrm{~g}$ ) sur centrifugeuse Mistral 6L. Le suivi de la solubilité des protéines était réalisé par détermination de l'extrait sec du surnageant. La fraction ultrafiltrable était séparée par ultrafiltration des échantillons sur membrane conique Centriflo CF 25 - Amicon (seuil de coupure $\mathrm{PM}=25000)(1 \mathrm{~h}-500 \mathrm{~g})$. La teneur en calcium fixé était déterminée par différence entre calcium total et calcium ultrafiltrable.

Déterminations analytiques : la détermination de l'extrait sec était réalisée par dessiccation de $2 \mathrm{~g}$ de solution sur dessiccateur à Infra-rouge Mettler LP 15 couplé à une balance électronique Mettler PC 220 à $160^{\circ} \mathrm{C}$ (Détermination à $\pm 0,1 \%$ ).

Le dosage du calcium était réalisé par spectrophotométrie d'absorption atomique sur appareil Varian AA 1275, selon le protocole de Murthy et RheA (1967) modifié par Brulé et al. (1974).

$\mathrm{La}$ viscosité des solutions était étudiée à $25^{\circ} \mathrm{C}$ à l'aide d'un viscosimètre rotatif à cylindres coaxiaux Rheomat 30 Contraves sur cellule MSO.

\section{Résultats}

\section{A. Solubilité}

Les essais étaient réalisés sur des solutions de caséinate et paracaséinate à $80 \mathrm{~g}$ de protéines par 1 pour $3 \mathrm{pH}(6,6-6,0-5,4)$ à une force ionique initiale de 0,036 , et 3 forces ioniques initiales $(0,077-0,198-0,376$ : correspondant à $3-10-20 \mathrm{~g} / 1 \mathrm{de} \mathrm{NaCl})$ à pH 6,6; des quantités croissantes de chlorure de calcium étaient ajoutées jusqu'à l'apparition d'un précipité. 
L'addition de faibles quantités de calcium n'altérait pas la solubilité des caséinate et paracaséinate. Par contre, l'accroissement de la teneur en calcium du milieu entraînait une chute de solubilité allant jusqu'à la floculation, rapide pour le paracaséinate, plus progressive pour le caséinate. Ceci est illustré par la figure 1. La précipitation des paracaséines débutait pour une teneur en calcium critique supérieure à $800 \mathrm{mg} / 1$ pour $\mathrm{pH} 6,6$ et 6,0 alors qu'à $\mathrm{pH} 5,4$ cette teneur critique en calcium était de l'ordre de $200 \mathrm{mg} / \mathrm{l}$. La floculation du caséinate débutait pour une quantité de calcium total au moins égale à $1800 \mathrm{mg} / 1$ à $\mathrm{pH} 6,6$; à $\mathrm{pH} 6,0$ et 5,4 la solubilité était affectée

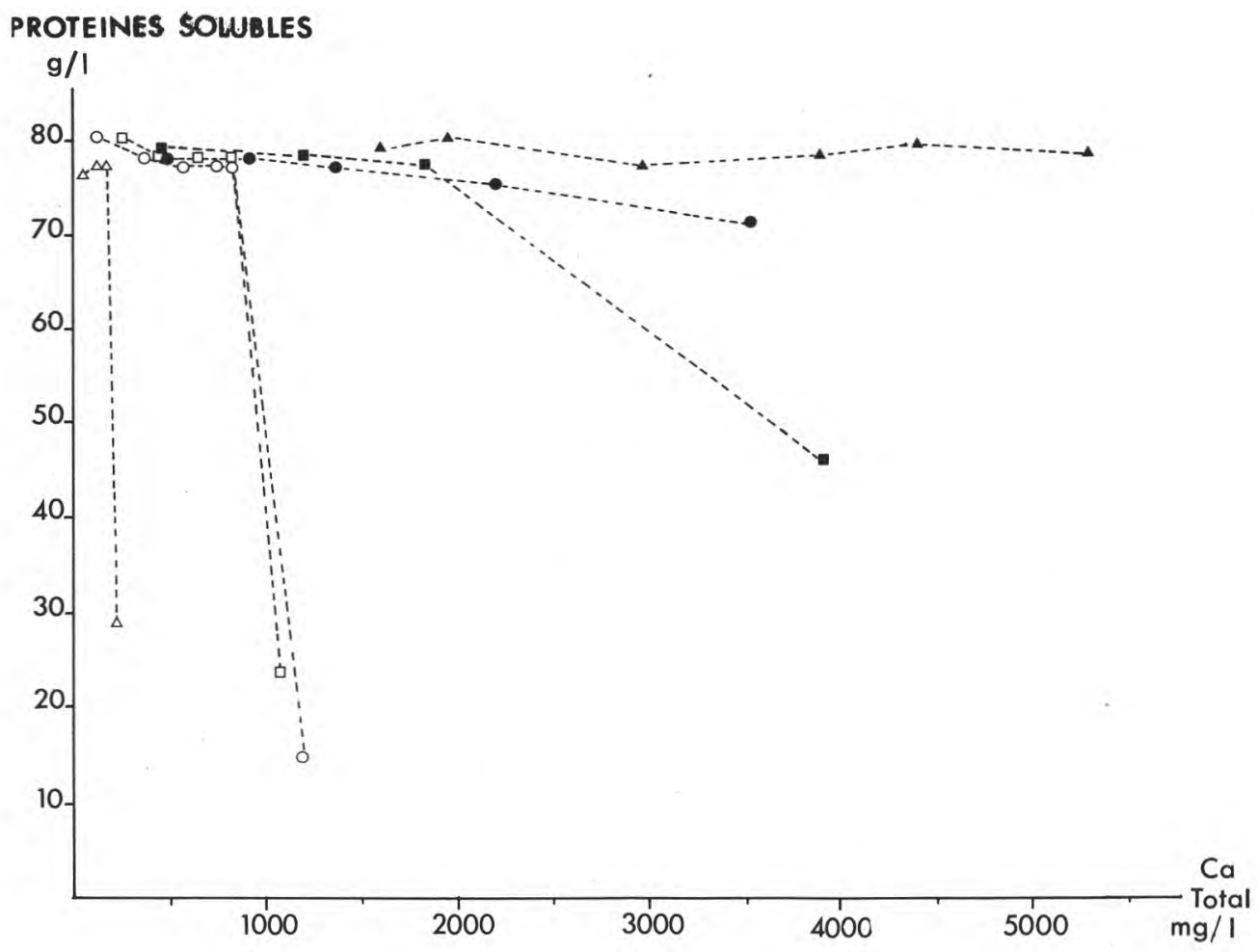

FIG. 1

Solubilité $d u$ caséinate et paracaséinate en solution à $80 \mathrm{~g} / \mathrm{l}, \mu=0,036$, à trois $p H$ différents en fonction de la concentration en calcium. Caséinate : ( $)$ pH 6,$6 ;(\bullet) p H 6,0 ;(\Delta) p H 5,4$. Paracaséinate: (口) $p H 6,6 ;(0) p H 6,0 ;(\triangle) p H ~ 5,4$.

Solubility of caseinate and paracaseinate for solutions at $80 \mathrm{~g} / \mathrm{l}, \mu=0.036$, at three different $\mathrm{pHs}$ as a function of calcium concentration. Caseinate : (1) $p H$

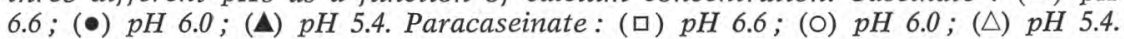


pour des teneurs en calcium respectivement supérieures à 2200 et $5300 \mathrm{mg} / 1$.

L'augmentation de la force ionique initiale par addition de $\mathrm{NaCl}$, avec maintien du $\mathrm{pH}$ à 6,6 , retardait la précipitation lors de l'addition de calcium. Dans le cas du paracaséinate, il y avait floculation pour des teneurs voisines de $1000,1400,1800 \mathrm{mg} / 1$ pour des solutions contenant respectivement $3 \mathrm{~g} / \mathrm{l}, 10 \mathrm{~g} / \mathrm{l}, 20 \mathrm{~g} / 1 \mathrm{de} \mathrm{Nacl}$ (fig. 2). Pour le caséinate, cette floculation commençait vers $2100 \mathrm{mg} / 1$ quelle que soit la quantité de $\mathrm{NaCl}$ ajouté.

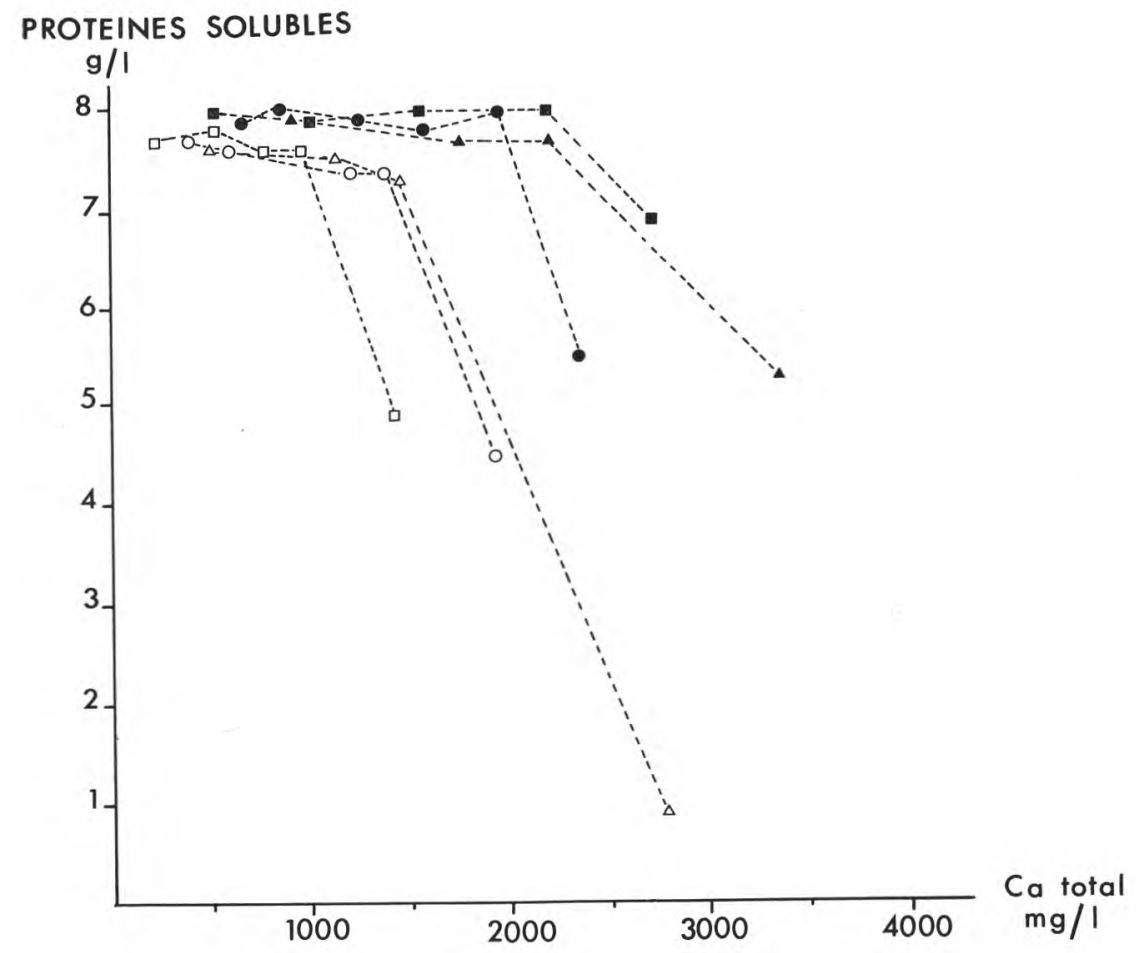

FIG. 2

Solubilité $d u$ caséinate et paracaséinate en solution à $80 \mathrm{~g} / \mathrm{l}, \mathrm{pH} 6,6$, à trois forces ioniques différentes en fonction de la concentration en calcium. Caséinate: (匹) $\mu=0,077 ;(\bullet) \mu=0,198 ;(\mathbf{\Delta}) \mu=0,376$. Paracaséinate : (口) $\mu=0,077$; (0) $\mu=0,198 ;(\triangle) \mu=0,376$.

Solubility of caseinate and paracaseinate for solutions at $80 \mathrm{~g} / \mathrm{l}, \mathrm{pH} 6.6$ at three different ionic strengths as a function of calcium concentration. Caseinate : (৫) $\mu=0.077 ;(\bullet) \mu=0.198 ;(\Delta) \mu=0.376$. Paracaseinate : (口) $\mu=0.077$; (0) $\mu=0.198 ;(\triangle) \mu=0.376$. 


\section{B. Affinité}

Lors de l'addition de faibles quantités de calcium aux solutions de caséinate et paracaséinate de sodium, la plus grande partie se fixait aux protéines, le reste étant soluble. La proportion de calcium fixé diminuait lors de l'augmentation des teneurs en calcium total : à $\mathrm{pH} 6,6$ pour des solutions à $80 \mathrm{~g} / 1$ de caséinate et des teneurs en calcium total de 440 et $1825 \mathrm{mg} / \mathrm{l}$, respectivement 89 et $57 \%$ du calcium étaient fixés. Dans ces conditions de $\mathrm{pH}$, de concentration en protéines et pour une force ionique initiale de 0,036, la quantité maximale de calcium fixé avant précipitation était de $13,5 \mathrm{mg} / \mathrm{g}$ de caséinate et $6,8 \mathrm{mg} / \mathrm{g}$ de paracaséinate soit 7,8 et 5,5 moles de calcium par mole de protéines. Le poids moléculaire moyen des caséines monomères a été pris égal à 23167 (HoLt, 1982).

L'augmentation de la teneur en caséinate des solutions de 7 à $111 \mathrm{~g} / \mathrm{l}$ (force ionique initiale de 0,002 à 0,026 ) (tab. 1) réduisait la quantité maximale de calcium fixé de 14,8 à 6,0 moles de $\mathrm{Ca}$ par mole de protéine. Un effet semblable était observé avec le paracaséinate.

La teneur en calcium fixé avant précipitation régressait également lors de la baisse du $\mathrm{pH}$ de 6,6 à 5,4 (tabl. 1) ; elle atteignait, à ce dernier $\mathrm{pH}, 3,5$ et 0,8 moles de calcium par mole de caséinate et paracaséinate respectivement.

Lors de l'augmentation de la force ionique par addition de $\mathrm{NaCl}$ (tabl. 1), la teneur maximale en calcium fixé s'abaissait de 7,8 à 5,7 moles de calcium par mole de caséinate pour $\mu=0,077$ et $\mu=0,376$. Pour les solutions de paracaséinate dans les mêmes conditions, cette teneur était peu variable, voisine de 5,5 moles de $\mathrm{Ca}$ par mole de paracaséinate.

\section{Viscosité}

Une étude préliminaire sur une gamme de vitesse de cisaillement $(\dot{\gamma})$ s'étalant de 111 à $1764 \mathrm{~s}^{-1}$ avait montré que le comportement rhéologique des solutions de caséinate et de paracaséinate à $80 \mathrm{~g} / \mathrm{l}$, ayant de faibles teneurs en calcium, était newtonien $\left(\mathrm{T}=25^{\circ} \mathrm{C}\right)$. Celui des solutions de paracaséinate à teneurs en calcium élevées était rhéofluidifiant. $\mathrm{La}$ viscosité d'une telle solution diminuait de $19,4 \mathrm{mPa}$.s pour $\dot{\gamma}=151 \mathrm{~s}^{-1}$ à $14,0 \mathrm{mPa}$.s pour $\dot{\gamma}=1764 \mathrm{~s}^{-1}$ (solution à $80 \mathrm{~g} / \mathrm{l}$ - teneur calcique : $1000 \mathrm{mg} / \mathrm{l}-\mathrm{pH}=6,6-\mu=0,036)$.

Les mesures de viscosité étaient réalisées dans la suite de nos études, sur des solutions de caséinate et paracaséinate à $80 \mathrm{~g} / \mathrm{l}$, à la même température $\left(25^{\circ} \mathrm{C}\right)$ et à une vitesse de cisaillement de $517 \mathrm{~s}^{-1}$ où le comportement des solutions était quasiment newtonien. 


\section{TABLEAU 1}

Teneur en calcium fixé lors du début de précipitation pour des solutions de caséinate et paracaséinate à différentes teneurs en protéines, à différents $\mathrm{pH}$, à différentes forces ioniques (en moles de Ca/mole de caséinate ou de paracaséinate)

Bound calcium content at precipitation for caseinate and paracaseinate solutions for different protein contents, for differents $\mathrm{pHs}$, for different ionic strengths (in calcium moles/caseinate or paracaseinate mole).

\begin{tabular}{|c|c|c|c|}
\hline $\begin{array}{c}\text { Teneur en } \\
\text { caséinate } \\
\text { g/l }\end{array}$ & $\mathrm{pH}$ & $\begin{array}{l}\text { Force ionique } \\
\text { initiale }\end{array}$ & $\begin{array}{l}\text { Moles } \mathrm{Ca} / \mathrm{mole} \\
\text { caséinate }\end{array}$ \\
\hline $\begin{array}{r}7 \\
21 \\
111\end{array}$ & $\begin{array}{l}7,1 \\
7,0 \\
6,7\end{array}$ & $\begin{array}{l}0,002 \\
0,006 \\
0,026\end{array}$ & $\begin{array}{r}14,8 \\
7,2 \\
6,0\end{array}$ \\
\hline $\begin{array}{l}80 \\
80 \\
80\end{array}$ & $\begin{array}{l}6,6 \\
6,0 \\
5,4\end{array}$ & $\begin{array}{l}0,036 \\
0,036 \\
0,036\end{array}$ & $\begin{array}{l}7,8 \\
7,2 \\
3,5\end{array}$ \\
\hline $\begin{array}{l}81 \\
80 \\
80\end{array}$ & $\begin{array}{l}6,7 \\
6,7 \\
6,7\end{array}$ & $\begin{array}{l}0,077 \\
0,198 \\
0,376\end{array}$ & $\begin{array}{l}7,8 \\
6,1 \\
5,7\end{array}$ \\
\hline $\begin{array}{l}\text { Teneur en } \\
\text { paracaséinate } \\
\mathrm{g} / \mathrm{l}\end{array}$ & $\mathrm{pH}$ & $\begin{array}{c}\text { Force ionique } \\
\text { initiale }\end{array}$ & $\begin{array}{c}\text { Moles } \mathrm{Ca} / \text { mole } \\
\text { paracaséinate }\end{array}$ \\
\hline $\begin{array}{r}7 \\
20\end{array}$ & $\begin{array}{l}7,1 \\
6,7\end{array}$ & $\begin{array}{l}0,002 \\
0,006\end{array}$ & $\begin{array}{r}12,8 \\
7,9\end{array}$ \\
\hline- & - & - & - \\
\hline $\begin{array}{l}77 \\
80 \\
76\end{array}$ & $\begin{array}{l}6,6 \\
6,0 \\
5,4\end{array}$ & $\begin{array}{l}0,036 \\
0,036 \\
0,036\end{array}$ & $\begin{array}{l}5,5 \\
3,4 \\
0,8\end{array}$ \\
\hline $\begin{array}{l}80 \\
77 \\
78\end{array}$ & $\begin{array}{l}6,7 \\
6,7 \\
6,7\end{array}$ & $\begin{array}{l}0,077 \\
0,198 \\
0,376\end{array}$ & $\begin{array}{l}5,2 \\
5,3 \\
5,7\end{array}$ \\
\hline
\end{tabular}

L'addition de chlorure de calcium, jusqu'à une teneur totale de $585 \mathrm{mg}$ de $\mathrm{Ca} / \mathrm{l}$, à une solution de caséinate à $\mathrm{pH} \mathrm{6,6} \mathrm{(fig.} \mathrm{3),} \mathrm{entraînait}$ une chute de la viscosité de $15,7 \mathrm{mPa}$.s à $11,3 \mathrm{mPa}$.s. La viscosité s'élevait ensuite à $12,7 \mathrm{mPa}$.s lorsque la teneur en calcium atteignait $815 \mathrm{mg} / 1$ pour chuter finalement à $3,7 \mathrm{mPa} . \mathrm{s}$ lorsque la teneur en calcium s'élevait à $1825 \mathrm{mg} / \mathrm{l}$. A pH 5,4 on retrouvait la même allure de courbe bien que celle-ci soit plus aplatie. Pour un caséinate à 


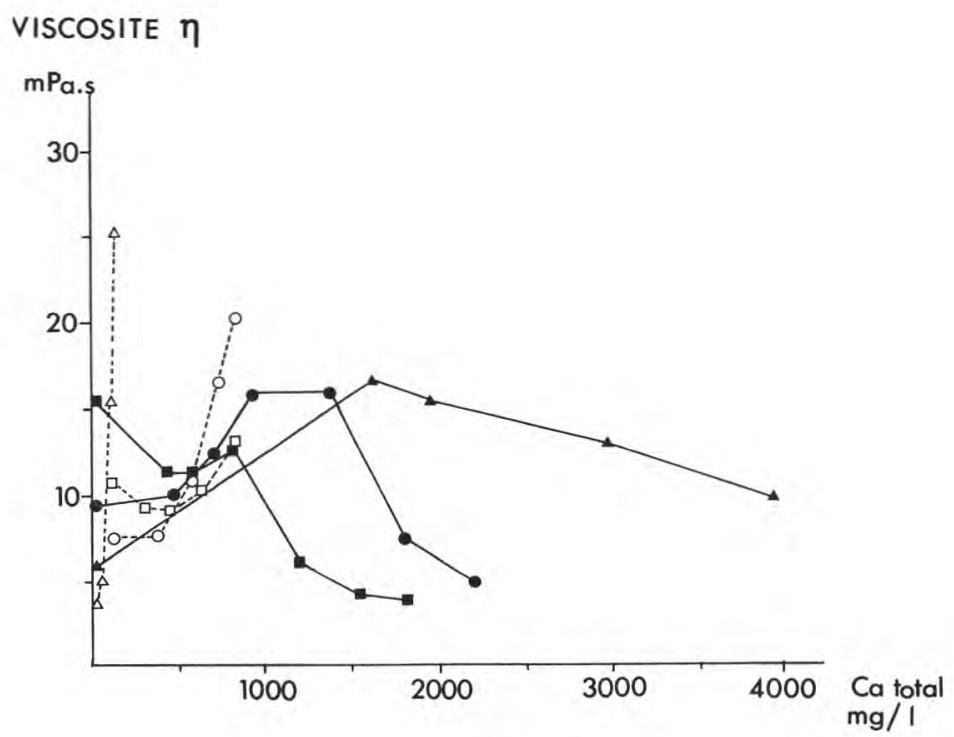

FIG. 3

Viscosité des solutions de caséinate et paracaséinate $\left(80 \mathrm{~g} / \mathrm{l}-\mathrm{T}=25^{\circ} \mathrm{C}-\dot{\gamma}=\right.$ $517 \mathrm{~s}^{-1}-\mu=0,036$ ) pour trois $\mathrm{pH}$ en fonction de la teneur en calcium total.

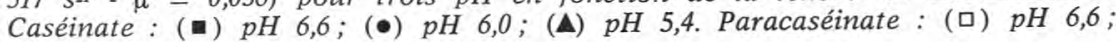
(O) $p H 6,0 ;(\triangle) p H 5,4$.

Viscosity of caseinate and paracaseinate solutions $\left(80 \mathrm{~g} / \mathrm{l}-\mathrm{T}=25^{\circ} \mathrm{C}-\dot{\gamma}=517 \mathrm{~s}^{-1}\right.$. $\mu=0,036)$ at three pHs as a function of total calcium. Caseinate ( $)$ pH 6.6 ;

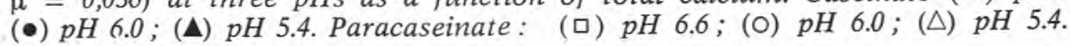

$\mathrm{pH} 5,4$ et une teneur en calcium égale à $800 \mathrm{mg} / 1$ on observait une gélification des solutions; ce comportement n'apparaissait plus pour des quantités ajoutées supérieures.

En augmentant la force ionique par addition de chlorure de sodium, la viscosité initiale des solutions de caséinate augmentait ; elle passait de 15,4 à 17,3 puis à $22,8 \mathrm{mPa}$.s pour 3,10 et $20 \mathrm{~g} / 1$ de $\mathrm{NaCl}$. Les courbes représentées sur la figure 4 ont une allure semblable à celles obtenues à $\mathrm{pH}$ 6,6 avec toutefois une viscosité supérieure atteignant un maximum de $37,6 \mathrm{mPa}$.s pour $20 \mathrm{~g} / 1 \mathrm{de} \mathrm{NaCl}$ et une teneur calcique de $1360 \mathrm{mg} / \mathrm{l}$.

Les paracaséinates avaient un comportement différent : la viscosité diminuait tout d'abord : à $\mathrm{pH}$ 6,6 elle passait de 10,8 $\mathrm{mPa} . \mathrm{s}$ pour $110 \mathrm{mg}$ de $\mathrm{Ca} / \mathrm{l}$ à $9,0 \mathrm{mPa}$.s pour $455 \mathrm{mg}$ de $\mathrm{Ca} / \mathrm{l}$; ensuite cette viscosité augmentait pour atteindre $13,1 \mathrm{mPa} . \mathrm{s}$ à $830 \mathrm{mg} / \mathrm{l}$. L'accroissement final de viscosité avant la coagulation était d'autant plus rapide que le pH était bas (fig. 3) : une augmentation de viscosité de $10 \mathrm{mPa}$.s était obtenue par une augmentation de concentration en calcium de $200 \mathrm{mg} / 1$ à pH 6,6 et de $20 \mathrm{mg} / 1$ à pH 5,4. 
Pour une même teneur en calcium, la viscosité était d'autant plus élevée que la force ionique était importante (fig. 4).

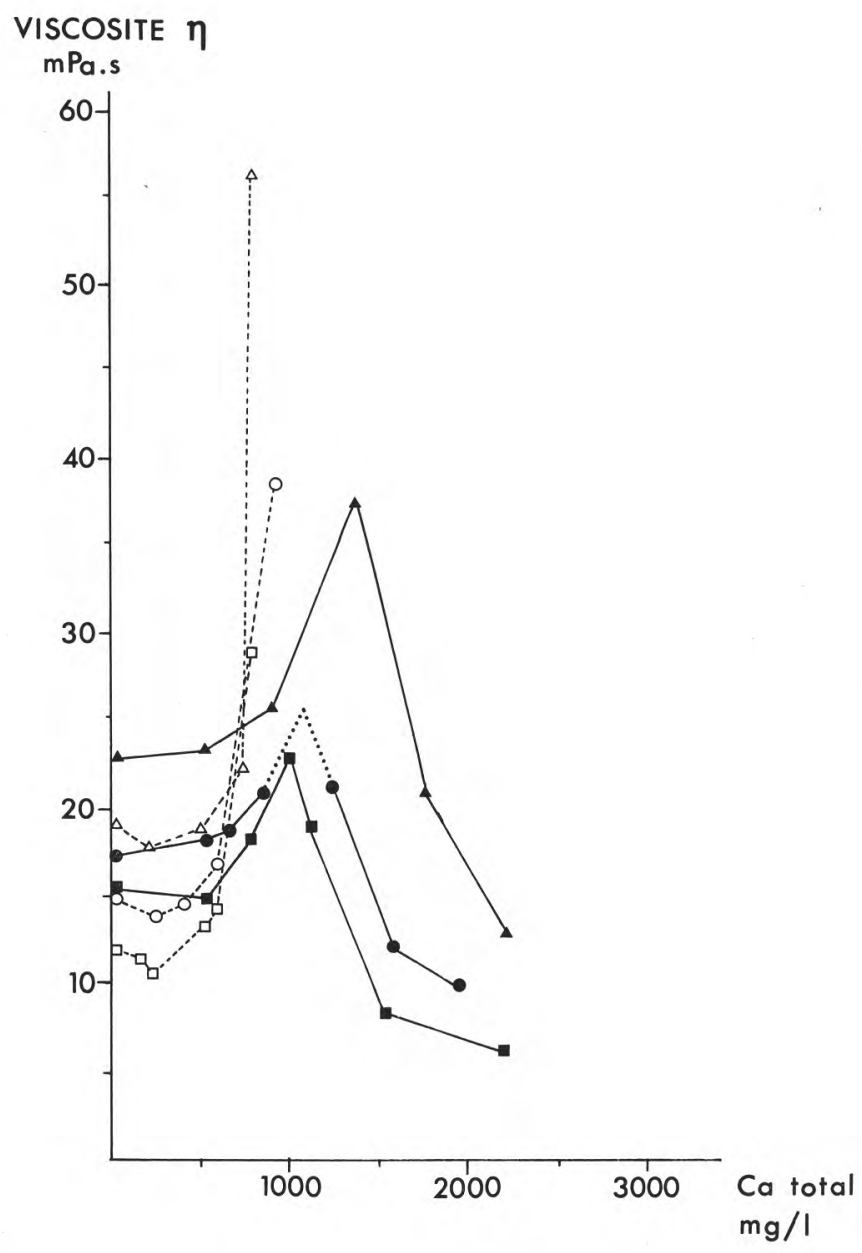

FIG. 4

Viscosité des solutions de caséinate et paracaséinate $\left(80 \mathrm{~g} / \mathrm{l}-\mathrm{pH} 6,6-\mathrm{T}=25^{\circ} \mathrm{C}\right.$ $\dot{\gamma}=517 \mathrm{~s}_{-}^{-1}$ ) pour trois forces ioniques en fonction de la teneur en calcium total. Caséinate : (⿴囗十) $\mu=0,077 ;(\bullet) \mu=0,198 ;(\Delta) \mu=0,376$. Paracaséinate : (口) $\mu=0,077$;

(O) $\mu=0,198$;

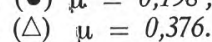

Viscosity of caseinate and paracaseinate solutions $\left(80 \mathrm{~g} / \mathrm{l}-\mathrm{pH} 6.6-\mathrm{T}=25^{\circ} \mathrm{C}\right.$ $\left.\dot{\gamma}=517 \mathrm{~s}^{-1}\right)$ at three ionic strengths as a function of total calcium. Caseinate :

(घ) $\mu=0.077 ;(\bullet) \mu=0198 ;(\mathbf{\Delta}) \mu=0.376$. Paracaseinate : (ㅁ) $\mu=0.077$; (0) $\mu=0.198 ;(\triangle) \mu=0.376$. 


\section{Discussion}

Les solutions de caséinate et paracaséinate de sodium (ou d'autres ions monovalents) forment le plus souvent des solutions colloïdales stables composées de submicelles en suspension. Lors de l'addition de calcium à ces solutions, nous avons vu que la plus grande partie se fixait aux caséines.

$\mathrm{Au}$ point critique (seuil de solubilité) l'examen des résultats rassemblés dans le tableau 1 et dans la figure 1 montre que $13,5 \mathrm{mg}$ de calcium sont fixés pour $1 \mathrm{~g}$ de caséinate et $9,4 \mathrm{mg}$ de Ca pour $1 \mathrm{~g}$ de paracaséinate pour des solutions à $80 \mathrm{~g} / 1$ de protéines (force ionique initiale $=0,036-\mathrm{pH}=6,6)$; dans des conditions voisines, le lait contient $29,3 \mathrm{mg}$ de Ca fixé pour $1 \mathrm{~g}$ de caséine $(\mu=0,1-\mathrm{pH}$ $=6,7)$; cette valeur très supérieure est due au pouvoir complexant plus élevé découlant de la présence de phosphate minéral au sein de la micelle de caséine,

Etant donné l'affinité pour le calcium, des caséines $\alpha_{S_{1}}, 4$ fois supérieure à celle de la caséine $\beta$, dans des conditions voisines de celles de nos expériences (Brulé et Fauquant, 1982), il est probable que le calcium ajouté aux solutions de caséinate et paracaséinate se fixe préférentiellement sur les résidus phosphoséryles des caséines $\alpha_{\text {s, }}$ puis sur ceux de la caséine $\beta$. La fixation au niveau des groupements carboxyles est probablement plus limitée. De ce fait, l'ion calcium forme en majorité un complexe soit au niveau des 2 acidités d'une phosphosérine soit entre les acidités de 2 résidus distincts (GRESH, 1980). Il est également probable qu'un équilibre existe entre ces 2 formes. Mais le nombre de moles de calcium fixé par mole de caséinate $(\mathrm{pH} 6,6-\mu=0,036)$ étant égal à 7,8 soit une valeur supérieure à celle du nombre moyen de sites phosphosériques (voisin de $6,5)$, un certain nombre d'ions calcium sont donc probablement liés aux groupements carboxyliques.

Les paracaséines ne fixant, dans les mêmes conditions, que 5,5 moles de calcium par mole de protéine, on peut en conclure que la précipitation se produit avant saturation totale des sites chélateurs de calcium.

A partir des submicelles présentes dans la solution, l'addition de calcium amène à une constitution progressive de micelles qui, dans le cas du caséinate, sont stabilisées par la caséine $\chi$ et donc ne précipitent pas, sauf pour des quantités de calcium ajouté élevées. Dans le cas du paracaséinate il y a agrégation rapide des micelles donc précipitation, due à l'absence de caséine $x$. Cette constitution progressive peut être confirmée par l'étude de la viscosité. Une première phase de fixation intramoléculaire du calcium se traduirait par des réductions de la charge nette des protéines et donc de leur hydratation. Cette dernière qui est de 3 et $7 \mathrm{~g}$ d'eau par $\mathrm{g}$ de 
caséines $\alpha_{\mathbb{S}_{1}}$ et $\beta$ natives passerait à 1,7 et $1,9 \mathrm{~g}$ par $\mathrm{g}$ de caséines en présence de calcium (SWAISGood, 1982). Cette diminution d'hydratation entraînerait une chute de la viscosité. La seconde phase se traduirait par la formation de liaisons intermoléculaires, donc par l'agrégation des submicelles, ce qui augmenterait la viscosité. Dans le cas des solutions de caséinate, la présence de submicelles riches en caséines $x$ limiterait la taille des micelles, compte tenu de leur faible teneur en phosphosérines; ce phénomène aurait pour effet d'améliorer la stabilité en milieu calcique et de diminuer la viscosité. Ces résultats sur l'évolution de la viscosité confirment ceux de TOWLER (1974).

La diminution des quantités de calcium fixé par le caséinate et le paracaséinate, consécutive à un abaissement de $\mathrm{pH}$, est due à la régression d'ionisation des phosphosérines. Expérimentalement, nous avons trouvé que les $2 \mathrm{pK}$ du groupement phosphate de la phosphosérine libre étaient égaux à 2,6 et 6,0 .

Lorsque le $\mathrm{pH}$ est abaissé à 5,4 une des deux fonctions acide des phosphosérines n'est plus capable de chélater le calcium; le nombre de sites de fixation est donc inférieur : pour le caséinate la quantité de calcium fixé passe de 7,8 moles de $\mathrm{Ca} / \mathrm{mole}$ de caséinate pour $\mathrm{pH} 6,6$ à 3,5 moles de $\mathrm{Ca} /$ mole de caséinate pour $\mathrm{pH} 5,4$. Le calcium, à pH 5,4, aura donc tendance à créer de préférence des liaisons intermoléculaires qui entraînent la formation plus rapide de micelles et donc l'augmentation de la viscosité.

De plus la baisse du pH entraîne une diminution de la charge nette du caséinate, qui le rend moins hydrophile donc plus précipitable (Mac MAHON et BRoWN, 1984).

La gélification d'une solution de caséinate ( $80 \mathrm{~g} / 1-\mu=0,085)$ à $\mathrm{pH} 5,4$ se produit pour une teneur en calcium total de $800 \mathrm{mg} / 1$ correspondant à $2,6 \mathrm{mg}$ de $\mathrm{Ca}$ fixé par g de caséinate; la quantité maximale pouvant être fixée à ce $\mathrm{pH}$ étant de $6,1 \mathrm{mg} / \mathrm{g}$ de caséinate, nous sommes donc ici proches de la demi déminéralisation de ces protéines, ce qui correspond à un atome de calcium pour 2 sites, les sites réactifs étant probablement ceux permettant des liaisons intermoléculaires. L'absence de gélification constatée pour des teneurs en calcium supérieures peut être due à l'effet de la force ionique.

En présence de chlorure de sodium, le calcium se fixe en quantité plus faible au caséinate; la solution reste stable pour des teneurs en calcium ajouté plus élevées. Il y a compétition entre le calcium et le sodium au niveau de l'équilibre « Minéral + Protéine $\rightleftarrows$ Complexe Protéine-Minéral ».

La diminution de la teneur maximale en calcium fixé consécutive à l'augmentation de la concentration en protéines des solutions peut être due à une force ionique croissante résultant de l'apport de sodium par la poudre de caséinate $(14,2 \mathrm{~g}$ de $\mathrm{Na} / \mathrm{kg}$ de poudre). 
L'augmentation de la viscosité constatée lors d'un accroissement de la force ionique initiale de 0,077 à 0,376 peut être due à une diminution de l'énergie de liaison Ca-Phosphosérine quí entraîne la transition plus facile entre liaison intramoléculaire et liaison intermoléculaire. $\mathrm{La}$ teneur maximale fixée est semblable quelle que soit la force ionique pour les paracaséinates ce qui tendrait à prouver que la précipitation se produit quand un certain pourcentage des sites phosphosériques et carboxyliques est complexé par le calcium.

Ce travail met en évidence l'incidence de l'environnement ionique et des caractéristiques physico-chimiques du milieu, ainsi que l'importance de la caséine $x$, sur les propriétés fonctionnelles du caséinate de sodium. Les résultats obtenus devraient permettre de mieux mấtriser la fabrication et 1'utilisation des caséinates et d'envisager la mise au point de produits présentant des caractéristiques rhéologiques d'un grand intérêt pour l'industrie agro-alimentaire.

\title{
Remerciements
}

Nous tenons à exprimer nos remerciements à M. MAUBOIS pour l'aide et les nombreux conseils qu'il nous a apportés dans la rédaction de cette publication.

\section{Summary}

\author{
Comparative study of solubility and viscosity \\ of sodium caseinate and sodium paracaseinate solutions \\ with calcium
}

Interactions between sodium caseinate, sodium paracaseinate and calcium as also their consequences on solubility and viscosity were studied in aqueous solutions. The different solubilities of these two products depend on the calcium concentration, $\mathrm{pH}$ and ionic strength $(\mu)$. Precipitation begins for caseinate and paracaseinate $(80 \mathrm{~g} / 1)$ when calcium exceeds respectively 1800 and $900 \mathrm{mg} / 1$ (pH $6.6-\mu=0.036)$.

Maximum bound calcium content is, at $\mathrm{pH} 6.6$ equal to 7.8 and 5.5 calcium moles per caseinate or paracaseinate mole respectively. When ionic strength increases, bound calcium decreases slowly for caseinate and is constant for paracaseinate.

Variations in viscosity of caseinate and paracaseinate solutions $(80 \mathrm{~g} / \mathrm{l})$ with increasing amount of added calcium is different $(\mathrm{pH} 6.6$ temperature $=25^{\circ} \mathrm{C}$ - shear rate $\left.=517 \mathrm{~s}^{-1}-\mu=0,036\right)$ : from initial viscosities respectively equal to 16 and $11 \mathrm{mPa}$.s, after a decrease for both of them, viscosity increases to $13 \mathrm{mPa} . \mathrm{s}$, sharply for paracaseinate, but slowly for caseinate with a final decrease to $4 \mathrm{mPa} . \mathrm{s}$. ; $\mathrm{pH}$ and ionic strength also have an influence on viscosity.

Key words : Caseinate - Paracaseinate - Calcium - Solubility - Viscosity. 


\section{Références bibliographiques}

Brulé G., Fauquant J., 1982. Interaction des protéines du lait et des oligoéléments. Lait, 62, 323-331.

Brulé G., Maubois J.L., Fauquant J., 1974. Etude de la teneur en éléments minéraux des produits obtenus par ultrafiltration du lait sur membrane. Lait, $54,600-615$.

GRESH N., 1980. Intermolecular chelation of two serine phosphates by $\mathrm{Ca}^{2+}$ and $\mathrm{Mg}^{2+}$. A theoretical structural investigation. Biochim. Biophys. Acta, 597, 345-357.

HoLt C., 1982. Inorganic constituents of milk. III. The colloidal calcium phosphate of cow's milk. J. Dairy Res., 49, 29-38.

Mac MaHon D.J., BRown R.J., 1984. Composition, structure and integrity of casein micelles : A review. J. Dairy Sci., 67, 499-512.

Murthy G.K., RheA U., 1967. Determination of major cations in milk by atomic absorption spectrophotometry. J. Dairy Sci., 50, 313-317.

Nitschmann H.L., Lehmann W., 1947. Zum Problem der Labwirkung auf Casein. Helv. Chim. Acta, 30, 804.

Ono T., Kaminogawa S., Odagiri S., Yamauchi K., 1976. A study on the binding of calcium ions to $\alpha_{\mathbb{S}_{1}}$-casein. Agric. Biol. Chem., 40, 1717-1723.

Parker T.G., Dalgleish D.G., 1981. Binding of calcium to $\beta$-casein. J. Dairy Res., 48, 71-76.

Pierre A., Brulé G., 1981. Mineral and protein equilibria between the colloidal and soluble phases of milk at low temperature. J. Dairy Res., 48, 417-428.

Pierre A., Brulé G., Fauquant J., 1983. Etude de la mobilité du calcium dans le lait à l'aide du calcium 45. Lait, 63, 473-489.

Pyne L., Mac Gann T.C.A., 1960. The colloidal phosphate in milk. J. Dairy Sci., $27,9$.

SchmidT D.G., 1982. Association of caseins and casein micelle structure. In : Developments in Dairy Chemistry. I. Proteins. Edited by Fox P.F., Applied Science Publishers, London and New York, 61-86.

SwaISGOoD H.E., 1982. Chemistry of milk protein. In : Developments in Dairy Chemistry. I. Proteins. Edited by Fox P.F., Applied Science Publishers, London and New York, 1-59.

Towler C., 1974. Rheology of casein solutions. N.Z.J. Dairy Sci. Technol., 9, $155-160$. 